\title{
Loop Recession of the Recti Muscles
}

\author{
P. V. MILLS, ${ }^{*}$ T. J. HYPER $\dagger$ and G. R. DUFF* \\ Cardiff and Swansea
}

\begin{abstract}
Summary
Scleral penetration with a suture needle is a well recognised risk of the surgical treatment of strabismus and may result in serious intra-ocular complications. The risk should be reduced by loop recession, a procedure derived from the adjustable suture technique. In practice in many cases this operation has also been found to have other advantages compared to a conventional recession. The surgical details of loop recession are described and the results obtained in 62 patients reviewed.
\end{abstract}

In the mid 1970s Jampolsky ${ }^{1}$ re-introduced the adjustable suture technique for the surgical treatment of strabismus. This procedure is not strictly applicable to young children in whom suture adjustment during the early post-operative period cannot be performed with topical anaesthesia. However, it is evident that despite the lack of direct suturing of the rectus muscle tendon to the sclera, a firm adhesion between these two tissues is rapidly established, thus any suture adjustment needs to be effected within less than 24 hours of the operation. ${ }^{1.2,3}$

It is apparent that the same technique could be used in younger patients with the proviso that the suture would need to be knotted definitively at operation. Such a procedure was formerly described as a controlled, or guarded, tenotomy but had become obsolete, no doubt due, partly, to the apparent precision of the conventional recession but also to reactions to the suture material then available. The successful re-introduction of the adjustable suture technique suggested that a controlled tenotomy using modern suture material might prove an acceptable alternative to a rectus recession, not only for young children but in patients of all age groups. The adjustable suture technique described by Fells $^{2}$ in 1981 was slightly amended for use in the present study and the operation termed a loop recession. It is acknowledged that Gobin ${ }^{4}$ formerly applied similar terminology to a technique for producing an augmented recession of the medial rectus muscle.

\section{Surgical Technique}

An L-shaped limbal conjunctival incision was used. A double-armed 6/0 Dexon-Plus or coated Vicryl suture on $8 \mathrm{~mm}$ spatulated needle was inserted through the muscle tendon as described by Fells ${ }^{2}$ with a locked whip stitch at each edge of the tendon and an intermediate suture bite, not necessarily full thickness (Fig.). Following division of the tendon from the sclera, each end of the suture was passed through the original insertion twice. The two ends were tied following adjustment to ensure the recession corresponded with the planned measurement, using a double lock followed by two single locks. The conjunctiva was closed with a single suture of $6 / 0$ plain collagen, $6 / 0$ plain catgut or $8 / 0$ coated Vicryl, with the knot buried.

\footnotetext{
From: * University Hospital of Wales, Cardiff. † Singleton Hospital, Swansea.
}

Correspondence to: Mr. P. V. Mills, FRCS, University Hospital of Wales, Heath Park, Cardiff.

Presented at the Annual Congress of the Ophthalmological Society of the United Kingdom, April 1987. 


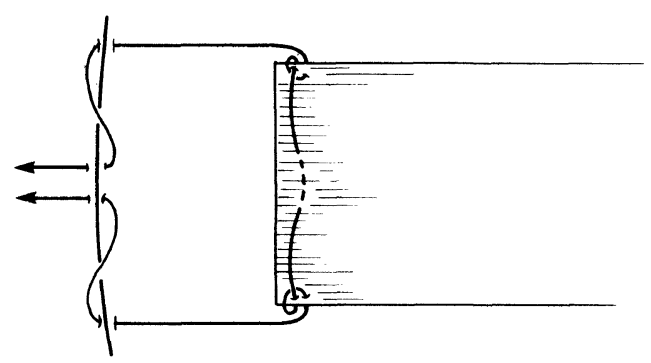

Fig. Diagram of a loop recession of a rectus muscle, showing suture placement in the muscle and the scleral insertion.

\section{Materials and Method}

Commencing in March 1985 at the University Hospital of Wales, loop recessions were performed instead of a conventional recession in a consecutive series of patients previously listed for a recession procedure. If a bilateral recession was scheduled, usually one eye was treated conventionally and the other by a loop recession. Patients were kept in hospital overnight and re-examined on the first post-operative day and thereafter at intervals of 1 week, 3 weeks and 7 weeks post-operatively. At these visits a full orthoptic assessment was undertaken together with clinical examination by at least one of the surgeons involved in the trial to assess the post-operative reaction.

In November 1985 the same surgical technique of loop recession was adopted at Singleton Hospital, Swansea, though post-operative follow-up was limited to clinical need.

The present view relates to a total of 62 patients, 31 from each centre, treated consecutively with a minimum follow-up period of 6 months. There were 28 male and 34 female patients. The age at operation ranged from 13 months to 62 years. Fiftysix patients had a concomitant squint and six an incomitant squint. The deviation was convergent in 44 patients, divergent in 13 and vertical in five. The operation of loop recession was performed on the medial rectus in 46 eyes, the lateral rectus in 17 eyes, the inferior rectus in 3 eyes and the superior rectus in 2 eyes.

The size of the loop recession used in each patient was the same as would have applied if a conventional recession operation had been performed, measured from the original insertion to the leading edge of the recessed tendon. In this series no attempt was made to utilise the technique of loop recession to achieve an augmented recession. Accordingly, the size of the loop recession varied from $4-5 \mathrm{~mm}$ for the medial recti and from $6-9 \mathrm{~mm}$ for the lateral recti. Loop recessions of the inferior recti ranged from $4.5-6 \mathrm{~mm}$, the larger recession being performed in a patient with dysthyroid ophthalmopathy and both superior rectus recessions measured $3.5 \mathrm{~mm}$.

In 6 patients the muscle treated by loop recession had been subjected to previous surgery. Five patients had a loop recession on one side and a conventional recession on the contralateral eye.

\section{Results}

The outcome of a loop recession in terms of ocular alignment and motility appeared no different to that characteristic of a conventional recession and there were no cases of persistent underaction. In the 31 patients operated upon at the University Hospital of Wales, underaction of the treated muscle was evident on the first post-operative day in 4 cases, as may occur with any recession procedure, but this fully resolved thereafter. In the whole group of 62 patients, seven were overcorrected but none to a degree meriting re-operation. One child being treated for a consecutive divergent squint became slightly convergent for near only and in one adult with bilateral aphakia a manifest convergent squint was converted to a latent divergence. In three of the 5 patients in whom a conventional recession was performed on one side and a loop recession on the contralateral eye, there was slightly more injection on the side of the loop recession at one week post-operatively but this disparity did not persist at 3 weeks.

The operation site of a loop recession was subsequently re-explored in only one patient. This child with a marked convergent squint originally had a conventional recession/resection operation on the right eye in October 1984 but due to undercorrection a $5 \mathrm{~mm}$ loop recession of the left medical rectus was performed in July 1985. As the angle of deviation was still unacceptable the left medial rectus was re-explored in January 1987 and found to show forward creep of approximately $2 \mathrm{~mm}$.

\section{Discussion}

Prior to commencing this study, it was felt a loop recession might have a number of potential advantages, and possibly a few disadvantages, compared to the usual recession operation (Table). Reference has been made to similar techniques in recent literature but 
Table. Loop Recession-Theoretical Considerations

\section{Potential Advantages}

Improved access to re-insertion site

Improved illumination of surgical field

Less retraction of conjunctiva/Tenons required

Less risk of snagging suture material

Improved visualisation despite any pooling of blood

Rotation of globe associated with circumferential suture placement avoided

Accurate alignment of muscle with original insertion

Reduced risk of scleral perforation

\section{Potential Disadvantages}

More suture material required

Possibly more frequent/intense suture reactions

Reliance on single knot

Forward creep with reduced effect/unpredictable results

no details of the surgical procedure or the results obtained are provided. ${ }^{5.6}$ One of the more compelling reasons seriously to consider a loop recession must be the risk of inadvertent needle penetration of the sclera in the conventional recession operation. In 1960 Havener and Kimball ${ }^{7}$ described four such cases, one of whom required enucleation of the eye following endophthalmitis. In the same year McLean, Galin and Baras reported characteristic retinal tears due to needle perforation at squint operations in 16 patients who presented within what the authors term 'a rather short period of time'. Ten years later Gottlieb and Castro ${ }^{9}$ detailed four further cases, including one with endophthalmitis and another blind from a retinal detachment. The same authors also found evidence of needle perforation in 6 ( 9.2 per cent) of 65 asymptomatic children partly ascribed to the former use of reverse cutting needles but the advent of spatulate needles has not eliminated the problem. In 1982 Salamon, Friberg and Luxenberg ${ }^{10}$ described two cases with post-operative endophthalmitis, one eventually requiring enucleation and in 1985 Apple and co-workers" presented the clinicopathologic findings of a child who had sustained bilateral perforation of the globe during medial rectus recession, one eye subsequently requiring enucleation due to painful phthisis bulbi. Ophthalmoscopic evidence of needle perforation was reported by Kaluzny and co-workers in $1977^{12}$ in 11 of 108 eyes (10.2 per cent) following recession operations and by Alio and Faci in $1984^{13}$ in 29 of 187 eyes (15.5 per cent) following the faden operation.

It is recognised that needle penetration of the sclera certainly can occur in resection operations when the sutures are inserted at the original muscle attachment site, as also applies in the technique of loop recession. In 1985 Basmadjian, Labelle and Dumas ${ }^{14}$ reported the development of a retinal detachment in 4 eyes from five to eight years after strabismus surgery, apparently the late result of needle perforation of the globe and in 2 eyes the perforation had occurred at the site of a resection operation. However, all the other cases traced in the literature in which serious sight damaging complications ensued, where adequate details are provided, relate to a rectus recession. Consequently, there would appear no reasonable doubt that a loop recession, in which the suture needle is not passed through the thin sclera posterior to the original insertion of the rectus muscle, incurs much less risk of inadvertant penetration of the globe than does a conventional recession.

The main theoretical objection to a loop recession concerns the possibility of excessive forward creep of the site of reattachment of the tendon to the sclera. In 1984 Climenhaga and Pearce ${ }^{15}$ reported experimental work on dogs, comparing the position of the muscle attachment 3 months after a conventional recession or a loop recession such as used in the present series of patients. They found that though the recessed muscles had minimally advanced in the majority of cases, the type of recession made little difference. However, the tendency to forward creep was much greater for the medial rectus than the lateral rectus muscles. This could well be relevant to our finding that an isolated loop recession of the medial rectus may prove relatively ineffective, significant forward creep being evident at re-operation in one such patient. Even so, the same operation performed on 3 other patients in this series produced a satisfactory result and isolated loop recessions of the inferior rectus were very effective in the management of dysthyroid ophthalmopathy.

With this one reservation, which only applies to a small minority of patients, we have come to appreciate the advantages of a loop recession (Table) which proved to be 
more than just theoretical and in most cases this operation took less time than the traditional procedure. The new technique proved particularly advantageous in small children, following previous surgery to the same muscle and to facilitate large recessions of the lateral recti. On the evidence of over 60 consecutive cases we are firmly of the opinion that a loop rectus recession is a safe and effective operation which we have now adopted as our standard technique in nearly all circumstances.

Mr. T. J. Hypher wishes to thank Mr. A. Pickering for kindly permitting the inclusion of four of his patients. The authors also acknowledge with gratitude the help of the orthoptic staff, particularly Miss J. Pinnick, Mrs. A. Hooper and Mrs. M. Watkins.

\section{References}

1 Jampolsky A: In 'Symposium on Strabismus'. Trans. New Orleans Academy of Ophthalmology. Helveston E M ed. St. Louis, Mosby 1978, 32149.

2Fells P. The use of adjustable sutures. Trans ophthalmol Soc UK 1981, 101: 279-83.

${ }^{3}$ Lee J, O'Day J, Fells, P: Early experience with adjustable squint surgery at Moorfields Eye Hospital with long-term follow-up. Trans ophthalmol Soc UK 1985, 104: 662-74.
${ }^{4}$ Gobin MH: Recession of the medial rectus muscle with a loop. Ophthalmologica 1968, 156: 25-7.

5 Mein $\mathrm{J}$ and Harcourt B: Diagnosis and management of ocular motility disorders. Oxford. Blackwell Scientific Publications 1985, 156-7.

${ }^{6}$ Willshaw HE: Rectus muscle surgery-how to do it. Trans ophthalmol Soc UK 1986, 105: 583-8.

${ }^{7}$ Havener WH and Kimball OP: Scleral perforation during strabismus surgery. Am J Ophthalmol 1960, 50: 807-8.

${ }^{x}$ McLean JM, Galin MA, Baras I: Retinal perforation during strabismus surgery. $A m \quad J$ Ophthalmol 1960, 50: 1167-9.

${ }^{y}$ Gottlieb F and Castro L: Perforation of the globe during strabismus surgery. Arch Ophthalmol 1970, 84: 151-7.

1" Salamon SM, Friberg TR, Luxenberg MN: Endophthalmitis after strabismus surgery. $A m J$ Ophthalmol 1982, 93: 39-41.

11 Apple DJ, Jones GR, Reidy JJ, Loftfield K: Ocular perforation and phthisis bulbi secondary to strabismus surgery. J Paed Oph and Strab 1985. 22: 184-7.

12 Kaluzny J, Ralcewicz H, Perlikiewicz-Kikielowa A: Eye fundus periphery after operation for squint. Klin Oczna 1977, 47: 557-58.

${ }^{13}$ Alio JL and Faci A: Fundus changes following faden operation. Arch Ophthalmol 1984, 102: 211-3.

${ }^{1+}$ Basmadjian G, Labelle P, Dumas J: Retinal detachment after strabismus surgery. Am J Ophthalmol 1975, 79: 305-9.

15 Climenhaga HW and Pearce WG: Adjustable sutures: experimental assessment of final muscle position. Can J Ophthalmol 1984, 19: 234-6. 